\title{
MORSE-SMALE ENDOMORPHISMS OF THE CIRCLE
}

\section{LOUIS BLOCK ${ }^{1}$}

ABSTRACT. The orbit structure of a continuously differentiable map $f$ of the circle is examined, in the case where the nonwandering set of $f$ is finite and hyperbolic. It is shown that there is a natural number $n(f)$ such that the period of any periodic point of $f$ is $n(f)$ times a power of 2 .

1. Introduction. It is well known (see [4]) that for a Kupka Smale diffeomorphism $f$ of the circle $S^{1}$ with $\Omega(f)$ finite, the following are true:

A. $\Omega(f)$ consists of periodic points.

B. The expanding and contracting periodic points alternate.

C. If $f$ is orientation preserving, all periodic points have the same period, and if $f$ is orientation reversing all periodic points have period one or two.

The purpose of this paper is to determine to what extent $A, B$, and $C$ are true for a Kupka $S_{m a l e}$ endomorphism $f$ of $S^{1}$ with $\Omega(f)$ finite. (To avoid unnecessary confusion caused by certain pathological cases, we also assume a generic property about the singularities of $f_{0}$ ) The results are stated in Theorems A, B, and C, following the necessary definitions.

We let $\operatorname{End}\left(S^{1}\right)$ denote the space of $C^{1}$ maps of $S^{1}$ into itself. Fix $f \in \operatorname{End}\left(S^{1}\right)$. A point $x \in S^{1}$ is said to be wandering if there is a neighborhood $N$ of $x$ in $S^{1}$ such that $f^{i}(N) \cap N=\varnothing, \forall i>0$. The set of points which are not wandering is called the nonwandering set and denoted $\Omega(f)$. A point $x \in S^{1}$ is called a periodic point if $f^{n}(x)=x$ for some natural number $n$. The minimum of $\left\{n \mid f^{n}(x)=x\right\}$ is called the period of $x$.

A periodic point $x$ of period $n$ is said to be contracting if $\left|D f^{n}(x)\right|<1$, and expanding if $\left|D f^{n}(x)\right|>1$. $f$ is said to be Kupka Smale if any periodic point of $f$ is expanding or contracting.

Received by the editors January 2, 1974 and, in revised form, February 25, 1974.

AMS (MOS) subject classifications (1970). Primary 58F 15.

Key words and phrases. Endomorphism, nonwandering set, periodic point.

1 This paper is a revision of a part of the author's thesis [1] completed at Northwestern University (and the Institut des Hautes Études Scientifiques) under the supervision of R. F. Williams. 
A point $x \in S^{1}$ is called a singularity of $f$ if $D f(x)=0 . x$ is said to be eventually periodic if $f^{m}(x)$ is periodic for some natural number $m$, or equivalently if $\operatorname{orb}(x)$ is finite, where $\operatorname{orb}(x)=\left\{f^{n}(x) \mid n \geq 0\right\}$.

We now define $M S\left(S^{1}\right)$ to be the set of $f \in \operatorname{End}\left(S^{1}\right)$ such that $\Omega(f)$ is finite, and:

(1) $f$ is Kupka $S$ male.

(2) No singularity of $f$ is eventually periodic.

For $f \in M S\left(S^{1}\right)$ we let $\Omega_{c}(f)$ (respectively $\Omega_{e}(f)$ ) denote the set of contracting (respectively expanding) periodic points of $f$.

We will prove the following:

Theorem A. If $f \in M S\left(S^{1}\right)$ then $\Omega(f)$ consists of periodic points.

Theorem B. Let $f \in M S\left(S^{1}\right)$, and card denote cardinality.

$$
\operatorname{card} \Omega_{e}(f) \leq \operatorname{card} \Omega_{c}(f) \leq \operatorname{card} \Omega_{e}(f)+1 .
$$

Equality (of card $\Omega_{e}(f)$ and card $\Omega_{c}(f)$ ) holds if and only if $f$ is onto. In the onto case the expanding and contracting periodic points alternate.

Theorem C. Let $f \in M S\left(S^{1}\right)$. There is a natural number $n(f)$ such that the period of any periodic point of $f$ is $n(f)$ times a power of 2. (Here we include $1=2^{0}$ as a power of 2.)

We conclude this section with a few remarks. First, suppose $f \in M S\left(S^{1}\right)$ is $C^{2}$ and satisfies the additional generic properties:

(3) All singularities of $f$ are nondegenerate (i.e. the second derivative is not zero).

(4) Orbits of distinct turning points are dis joint.

Then $f$ is structually stable (see [1] or [3]). In fact, the set of maps $f$ satisfying these properties can be classified up to topological conjugacy, by associating to each such $f$ a finite diagram consisting of certain eventually periodic points of $f$ and iterates of the singularities of $f$ (see [1] for details, or [2] where a special case is studied).

Second, since $x \in \Omega(f) \Rightarrow f(x) \in \Omega(f)$, it is obvious that $f \in M S\left(S^{1}\right)$ and $x \in \Omega(f)$ imply orb $(x)$ is finite. However this does not mean $x$ is periodic for endomorphisms. So Theorem $A$ is not immediate as it is in the diffeomorphism case.

Third, we note that one can construct (by induction) for any natural number $n$, a map $f_{n}$ in $M S\left(S^{1}\right)$ with periodic points of period $1,2,4, \cdots, 2^{n}$ (see [1] for details). Thus the statement in Theorem $C$ is essentially the most that can be said. 
Finally, we remark that Theorems $A, B$, and $C$ are true without the assumption that no singularity is eventually periodic. However, dropping this assumption makes a few of the proofs somewhat cumbersome, while add. ing little generality.

2. Proof of Theorem A. Let $f \in \operatorname{End}\left(S^{1}\right)$, and let $x$ be an expanding periodic point of period $n$. We let $W_{l}^{u}(x)$ denote the local unstable manifold of $x$, which is simply an open interval about $x$ on which $\left|D f^{n}\right|>1$, such that $f^{n}\left(W_{l}^{u}(x)\right)^{\prime} \supset W_{l}^{u}(x)$. We set $W^{u}(x)=\operatorname{orb}\left(W_{l}^{u}(x)\right)$, where orb $(A)$ is defined for any set $A$ by $\operatorname{orb}(A)=\bigcup_{n \geq 0} f^{n}(A)$.

We will use the following remark in the proof of Proposition 1 . If $g$ is a continuous map of $S^{1}$ into itself, and $I$ is a closed interval in $S^{1}$ with $g(I)$ ' $I$ and $g(I) \neq S^{1}$, then $g$ has a fixed point in $I$. This statement follows imme o diately from continuity (Rolle's theorem), but is false without the hypothes is $g(I) \neq S^{1}$.

Theorem A follows immediately from the following proposition.

Proposition 1. Suppose $f \in \operatorname{End}\left(S^{1}\right)$ is Kupka.Smale and no singularity of $f$ is eventually periodic. Suppose $y \in \Omega(F)$ is eventually periodic but not periodic. Then $y$ is a limit of periodic points.

Proof. By hypothes is there is an expanding periodic point $p$ and an integer $k>0 \mathrm{with} f^{k}(y)=p$. Let $V$ be any neighborhhod of $y$. By choosing $V$ smaller if necessary, we may assume that $f^{k}(V)$ is a neighborhood of $p$ in $W_{l}^{u}(p)$.

Note that $y \in \overline{W^{u}(p)}$ or else $y$ would be wandering. But since $\overline{W^{u}(p)}$ $W^{u}(p)$ is a finite invariant set, we have $y \in W^{u}(p)$. Hence $\exists y_{1} \in W_{l}^{u}(p)$ and $n>0$ with $f^{n}\left(y_{1}\right)=y$. Let $W$ be a closed interval about $y_{1}$ in $W_{l}^{u}(p)$ such that $f^{n}(W)$ is a neighborhood of $y$ in $V$. Then $f^{n+k}(W)$ is a neighborhood of $p$ in $W_{l}^{u}(p)$.

Now, there is a closed interval $K \subset f^{n+k}(W)$, and an integer $l>0$, such that $f^{l}(K)=W$. So, $f^{n+k+l}(K)=f^{n+k}(W)$, which is a proper closed interval containing $K$. Hence $K$ contains a periodic point, which implies that all iterates of $K$ contain periodic points. In particular, since $V \supset f^{n}(W)=$ $f^{n+l}(K), V$ has a periodic point. Since $V$ was arbitrary this completes the proof. Q.E.D.

\section{Proof of Theorem B.}

Lemma 2. Let $f \in M S\left(S^{1}\right)$ and let $p$ be an expanding periodic point of $f$. 
There does not exist $y \in\left(\overline{W^{u}(p)}-\operatorname{orb}(p)\right)$ with $p \in \operatorname{orb}(y)$.

Proof. Such an element $y$ would be nonwandering, but not periodic, a contradiction by Theorem A. Q.E.D.

We now make another definition. We will use the notation $[a, b]$ to denote the arc from $a$ to $b$ in which $b$ is in the counterclockwise direction from $a$. Let $f \in M S\left(S^{1}\right)$ 。 Let $p$ be an orientation preserving expanding fixed point of $f$. Set $W^{u}(p, c c)=\operatorname{orb}[p, b]$, where $b$ is a point in $W_{l}^{u}(p)$ in the counterclockwise direction from $p$, and set $W^{u}(p, c l)=\operatorname{orb}[a, p]$, where $a$ is a point in $W_{l}^{u}(p)$ in the clockwise direction from $p$. From the definition of $W_{l}^{u}(p)$, it follows that $W^{u}(p, c c)$ and $W^{u}(p, c l)$ are independent of the choices for $a$ and $b$. If $p$ is an orientation reversing expanding fixed point, define $W^{u}(p, c c)$ and $W^{u}(p, c l)$ by thinking of $p$ as an orientation preserving fixed point of $f^{2}$. Finally, if $p$ is an expanding periodic point of period $n$, define $W^{u}(p, c c)$ and $W^{u}(p, c l)$ by thinking of $p$ as a fixed point of $f^{n}$.

Proposition 3. Let $p$ be an expanding periodic point of $f \in M S\left(S^{1}\right)$ and let $I=\overline{W^{u}(p, c c)}$ or $I=\overline{W^{u}(p, c l)}$. Then $I$ is a proper subinterval of $S^{1}$ which contains another periodic point (besides $p$ ), and the closest periodic point to $p$ in $I$ is contracting.

Proof. By looking at a power of $f$, we may assume without loss of generality that $p$ is an orientation preserving fixed point. We may also assume that $I=\overline{W^{u}(p, c c)}$. If $I=S^{1}, \exists y \neq p$ in $W^{u}(p, c c)$ with $f(y)=p$. This contradicts Lemma 2. Hence $I$ is a proper subinterval of $S^{1}$. Let $I=[p, b]$.

We put an order $<$ on $I$ by identifying $I$ with a subinterval of the real line. If $f(b)=b$ then $b$ is a fixed point of $f$ in $I$. If not $f(b)<b$. Since $p$ is expanding, $\exists d \in W_{l}^{u}(p)$ in $[p, b]$ with $d<f(d)$. By continuity $f$ has a fixed point in $[p, b]$.

Let $c$ be the closest periodic point to $p$ in $I$. We must show that $c$ is contracting. Without loss of generality we may assume that $c$ is an orientation preserving fixed point. Suppose $c$ is expanding. $\exists l<c$, with $f(l)<l$. Hence there is a fixed point in $[d, l]$. This contradicts the fact that $c$ is the closest periodic point to $p$ in $I$. Hence $c$ is contracting. Q.E.D.

If $c$ is a contracting periodic point of period $n$ of $f \in \operatorname{End}\left(S^{1}\right)$, we define the stable manifold of $c$ by $W^{s}(c)=\left\{x \in S^{1} \mid c\right.$ is a limit point of orb $\left.(x)\right\}$. The component of $W^{s}(c)$ which contains $c$ is called the semilocal stable manifold of $c$, and is denoted by $\operatorname{slsm}(c)$.

Proposition 4. Let $c$ be a contracting periodic point of $f \in M S\left(S^{1}\right)$. If 
$\operatorname{slsm}(c) \neq S^{1}$, then one of the endpoints of $\operatorname{slsm}(c)$ is an expanding periodic point.

Proof. Let $E$ be the set of endpoints of $\operatorname{slsm}(c)$. $E$ has one or two ele ments and $f^{n}(E) \subset E$, where $c$ is of period $n$. Hence $f$ has a periodic point in $E$. We show that any periodic point $p \in E$ is expanding. Suppose $p$ is cono tracting. We may assume that $c$ and $p$ are orientation preserving fixed points, and $p$ is in the counterclockwise direction from $c$. Put an order $<$ on $[c, p]$ as in Proposition 3. $\exists a$ and $b$ in $(c, p)$ with $f(a)<a$ and $b<f(b)$. Hence there is a fixed point in $(c, p)$. This is a contradiction since $c$ is the only fixed point in $s \operatorname{lsm}(c)$. Q.E.D.

The following proposition follows almost immediately from the Lefschetz trace formula (see [6]).

Proposition 5. If $f \in M S\left(S^{1}\right)$ then the degree of $f$ is $0,+1$, or -1 . If the degree of $f$ is 0 , then card $\Omega_{c}(f)=$ card $\Omega_{e}(f)+1$. If the degree of $f$ is \pm 1 then card $\Omega_{c}(f)=$ card $\Omega_{e}(f)$.

Proposition 6. Let $f \in M S\left(S^{1}\right)$ be onto。 Then $\operatorname{card} \Omega_{e}(f)=\operatorname{card} \Omega_{c}(f)$ 。

Proof. Without loss of generality we may assume that all the periodic points of $f$ are orientation preserving fixed points. Suppose the statement is false. Then there are two contracting fixed points $c_{1}$ and $c_{2}$ such that the interval $\left(c_{1}, c_{2}\right)$ contains no fixed points. (The only other possibility is that $\Omega(f)$ consists of a single fixed sink $c$, but this would imply $f$ is not onto by Proposition 4.)

Let $I=\left[c_{1}, c_{2}\right]$. Pick points $t_{1} \in \operatorname{slsm}\left(c_{1}\right)$ and $t_{2} \in \operatorname{slsm}\left(c_{2}\right)$ in $I$, such that $f\left(t_{1}\right) \in\left(c_{1}, t_{1}\right)$ and $f\left(t_{2}\right) \in\left(t_{2}, c_{2}\right)$. Let $J=\left[t_{1}, t_{2}\right]$. Then $f(J) \supset\left[f\left(t_{2}\right)\right.$, $f\left(t_{1}\right)$. (For, if $f(J)$ did not conta in this interval, it would have to contain $\left[f\left(t_{1}\right), f\left(t_{2}\right)\right]$. Then $f(J) \supset J$ and $f(J)$ is a proper subinterval of $S^{1}$. Hence there is a fixed point in $J$, a contradiction.)

Let $\Omega_{e}(f)=\left\{e_{1}, \cdots, e_{n}\right\}$. There are points $k_{1}, \cdots, k_{n}$ in $J$ such that $f\left(k_{i}\right)=e_{i}$ for $i=1, \cdots, n$. Since $f$ is onto for each $i=1, \cdots, n$, we can find a sequence $\left(k_{i}^{-m}\right)$ with $k_{i}^{0}=k_{i}$ and $f\left(k_{i}^{-m}\right)=k_{i}^{-m+1} \forall m>0$. The sequence $\left(k_{i}^{-m}\right)$ must have a limit point, and a limit point of this sequence is clearly nonwandering. So to each $k_{i}$ we can assign an expanding fixed point $e_{j}$ such that $e_{j}$ is a limit point of the sequence $\left(k_{i}^{-m}\right)$. Define a map $T$ : $\left\{k_{1}, k_{2}, \cdots, k_{n}\right\} \rightarrow\left\{k_{1}, k_{2}, \cdots, k_{n}\right\}$ by $T\left(k_{i}\right)=k_{j}$, where $e_{j}$ is the chosen limit point of $\left(k_{i}^{-m}\right)$. Any map from a finite set into itself has a periodic 
point, so there is a subset of $\left\{k_{1}, k_{2}, \cdots, k_{n}\right\}$, say $\left\{k_{j_{1}}, \cdots, k_{j_{r}}\right\}$, such that $T\left(k_{j_{i}}\right)=k_{j_{i+1}}$ for $i=1, \cdots, r-1$ and $T\left(k_{j_{r}}\right)=k_{j_{1}}$.

Let $U$ be any neighborhood of $k_{j_{1}} \cdot T\left(k_{j_{r}}\right)=k_{j_{1}}$ means that $e_{j_{1}}$ is a limit point of $\left(k_{j_{r}}^{-m}\right)$. Now $f(U)$ is a neighborhood of $e_{j_{1}}$ so some iterate of $U$ contains $k_{j_{r}}$. Then $T\left(k_{j_{r-1}}\right)=k_{j_{r}}$ means $e_{j_{r}}$ is a limit point of $\left(k_{j_{r-1}}\right)$. So some iterate of $U$ contains $k_{j_{r-1}}$. It follows after $r-2$ more steps that an iterate of $U$ contains $k_{j_{1}}$ and hence intersects $U$. Since $U$ was arbitrary, $k_{j_{1}}$ is nonwandering. This is a contradiction and completes the proof. Q.E.D。

Theorem B now follows from Propositions 5 and 6 (and the fact that if $f$ is not onto then the degree of $f$ is 0 ). In view of Proposition 5, the following corollary is essentially a restatement of the content of Theorem B.

Corollary 7. If $f \in M S\left(S^{1}\right)$ and the degree of $f$ is 0 then $f$ is not onto.

\section{Proof of Theorem C.}

Proposition 8. Let $e$ and $c$ be adjacent expanding and contracting periodic points of $f \in M S\left(S^{1}\right)$ with c fixed. Then e is fixed by $f^{2}$.

Proof. Without loss of generality we may assume that there are no periodic points in $(e, c)$. Let $e_{1}$ be the closest point to $e$ in the counterclockwise direction from $e$, in orb $(e)$. We have two cases.

Case 1. $f(e) \neq e_{1}$. Then $f([e, c])$ contains $c$ and the point $f(e)$ which is not in $\left[e, e_{1}\right]$. Hence $\exists x \in(e, c)$ such that $f(x)=e$ or $f(x)=e_{1}$. In either case $e \in \operatorname{orb}(x)$, a contradiction by Lemma 2 and Proposition 3.

Case 2. $f(e)=e_{1}$. Note $\left[e, e_{1}\right] \subset W^{u}(e)$, because $f([e, c])$ is an interval containing $c$ and $e_{1}$, so $f([e, c]) \supset\left[c, e_{1}\right]$. If $f\left(e_{1}\right)=e$ we are done, so suppose $f\left(e_{1}\right) \neq e$. Then $f\left(\left[e, e_{1}\right]\right)$ contains $c$, and the point $f\left(e_{1}\right)$ is not in $\left[e, e_{1}\right]$. Hence $\exists y \in\left(e, e_{1}\right)$ such that $f(y)=e$ or $f(y)=e_{1}$. In either case $e \in \operatorname{orb}(y)$, a contradiction by Lemma 2 .

Proposition 9. Let $e$ and $c$ be adjacent expanding and contracting periodic points of $f \in M S\left(S^{1}\right)$ with e fixed. Then $c$ has period a power of 2 . (Here we include $1=2^{0}$ as a power of 2.)

Proof. Suppose not. Without loss of generality we may assume that there are no periodic points in $(e, c)$. Let $p$ be the closest periodic point to $e$, in the counterclockwise direction from $e$, which has period a power of 2 (there is such a $p$ by the proof of Proposition 3). Suppose $p$ is of period 
$k=2^{n}$. If we let $g=f^{2 k}$, then in the interval $[e, p], g$ has only two fixed points, $e$ and $p$, both of which are orientation preserving. It follows that $p$ is contracting. For if $p$ is expanding, then by the proof of Proposition 3, $p \in W^{u}(e, c c)$ and $e \in W^{u}(p, c l)$. This implies that there is a nonperiodic nonwandering point in $W^{u}(e, c c)$, a contradiction.

Let $b$ be the closest periodic point to $p$ in $(e, p)$. Then $b$ is expanding by the proof of Theorem $\mathrm{B}$, since $[b, p]$ is in $\operatorname{Im}(g)$. Under $g, p$ is a contracting fixed point, and $b$ is an expanding periodic point of period greater than 2. This contradicts Proposition 8. Q.E.D.

Theorem $C$ now follows from Propositions 8 and 9 and Theorem B.

\section{REFERENCES}

1. L. Block, Bifurcations on endomorphisms of $S^{1}$, Thesis, Northwestern University, Evanston, Ill., 1973.

2. L. Block and J. Franke, A classification of the structurally stable contracting endomorphisms of $S^{1}$, Proc. Amer. Math. Soc. 36 (1972), 597-602. MR 46 \#8264.

3. M. V. Jakobson, On smooth mappings of the circle into itself, Mat. Sb. 85 (127) (1971), 163-185= Math. USSR Sb. 14 (1971), 161-185. MR 44 \#7587.

4. M. Peixoto, Structural stability on two-dimensional manifolds, Topology 1 (1962), 101-120. MR 26 \#426.

5. M. Shub, Endomorphisms of compact differentiable manifolds, Amer. J. Math. 91 (1969), 175-199. MR 39 \#2169.

6. S. Smale, Differentiable dynamical systems, Bull. Amer. Math. Soc. 73 (1967), 747-817. MR 37 \#3598; erratum, p. 1593.

DEPARTMENT OF MATHEMATICS, UNIVERSITY OF FLORIDA, GAINESVILLE, FLORIDA 32611 